\title{
C-to-U editing and site-directed RNA editing for the correction of genetic mutations
}

\author{
Luyen Thi Vu, Toshifumi Tsukahara* \\ School of Materials Science, Japan Advanced Institute of Science and Technology (JAIST), Nomi City, Ishikawa, Japan.
}

\begin{abstract}
Summary Cytidine to uridine (C-to-U) editing is one type of substitutional RNA editing. It occurs in both mammals and plants. The molecular mechanism of $\mathrm{C}$-to- $\mathrm{U}$ editing involves the hydrolytic deamination of a cytosine to a uracil base. C-to-U editing is mediated by RNAspecific cytidine deaminases and several complementation factors, which have not been completely identified. Here, we review recent findings related to the regulation and enzymatic basis of C-to-U RNA editing. More importantly, when C-to-U editing occurs in coding regions, it has the power to reprogram genetic information on the RNA level, therefore it has great potential for applications in transcript repair (diseases related to thymidine to cytidine $(T>C)$ or adenosine to guanosine $(A>G)$ point mutations). If it is possible to manipulate or mimic $\mathrm{C}$-to- $\mathrm{U}$ editing, $\mathrm{T}>\mathrm{C}$ or $\mathrm{A}>\mathrm{G}$ genetic mutation-related diseases could be treated. Enzymatic and non-enzymatic site-directed RNA editing are two different approaches for mimicking C-to-U editing. For enzymatic site-directed RNA editing, C-to-U editing has not yet been successfully performed, and in theory, adenosine to inosine (A-to-I) editing involves the same strategy as $\mathrm{C}$-to- $\mathrm{U}$ editing. Therefore, in this review, for applications in transcript repair, we will provide a detailed overview of enzymatic site-directed RNA editing, with a focus on A-to-I editing and non-enzymatic sitedirected C-to-U editing.
\end{abstract}

Keywords: C-to-U editing, ${ }^{\mathrm{CV}} \mathrm{U}$-oligodeoxynucleotides, ${ }^{\mathrm{CNV}} \mathrm{K}$-oligodeoxynucleotides, APOBEC1, enzymatic site-directed RNA editing, non-enzymatic site-directed RNA editing

\section{Introduction}

Cytidine to uridine (C-to-U) RNA editing is a posttranscriptional process that modifies one or more cytidine $(\mathrm{C})$ nucleotides to uridine (U) nucleotides in transcript sequences, without changing the corresponding genomic sequences. C-to-U RNA editing involves various mechanisms and functions; the process can create start or stop codon and can alter encoded amino acids and splice site choices (1). C-to-U RNA editing was first reported in vertebrates for the mRNA encoding apolipoprotein B (apoB). The mechanism

Released online in J-STAGE as advance publication May 8, 2017.

*Address correspondence to:

Dr. Toshifumi Tsukahara, School Materials of Science, Japan Advanced Institute of Science and Technology (JAIST), 1-1 Asahidai, Nomi City, Ishikawa 923-1292, Japan.

E-mail: tukahara@jaist.ac.jp of apoB editing was later shown to involve hydrolytic deamination at the $\mathrm{C} 4$ position of the cytidine $(2,3)$. This conversion requires cis-acting elements (tripartite regulatory sequences) surrounding the edited cytidine and trans-acting elements (a multiprotein complex known as the editosome), which includes a catalytic cytidine deaminase and multiple auxiliary proteins (1). C-to-U RNA editing has also been reported in mitochondria and chloroplasts in higher plants (4). C-to-U RNA editing is conserved among species of flowering plants and usually occurs within regions of highly conserved amino acid sequences of mitochondrial proteins (5).

$\mathrm{C}$-to- $\mathrm{U}$ editing and adenosine to inosine (A-to-I) editing are two distinct types of substitutional RNA editing in mammals. Because substitutional RNA editing has powerful potential to recode point mutations, many studies have focused on manipulating and mimicking RNA editing; a therapeutic RNA editing approach was first proposed and applied in 1995. The goal of this 
approach is to correct mutated RNA sequences in order to treat genetic diseases caused by point mutations. Subsequently, several other studies have improved and developed this method, termed enzymatic sitedirected RNA editing. This innovative approach could potentially be applied for treating human diseases such as some neurological disorders by repairing adenosine or cytidine mutations in mRNA without changing the endogenous level of the mRNA target. Enzymatic sitedirected RNA editing includes not only C-to-U editing but also A-to-I editing. While enzymatic site-directed A-to-I RNA editing was successfully performed both in vitro and in cells, enzymatic site-directed C-to-U RNA editing has not yet been reported. Theoretically, both of these editing approaches employ the same strategy and the RNA editing machinery requires two main factors: complementary RNA sequences that can specifically bind to targeted sequences (guide RNA) and the editing enzyme that performs deamination. Moreover, nonenzymatic site-directed C-to-U editing was recently reported and has prompted great interest because it does not require the restrictive conditions dictated by sitedirected enzymatic RNA editing. Therefore, the present review focuses on enzymatic site-directed A-to-I RNA editing and non-enzymatic site-directed C-to-U RNA editing for correcting genetic diseases.

\section{C-to-U RNA editing in mammals}

Although various types of RNA editing occur in mammalian cells, deamination of adenosine to inosine (A-to-I) or cytidine to uridine (C-to-U) are the two major types of RNA editing $(6,7)$. A-to-I deamination has been observed at hundreds of thousands of sites and mostly occurs in non-coding and intronic regions, especially in targets containing Alu repeat sequences $(8,9)$. When A-to-I editing occurs in coding regions, it has most commonly been associated with the recoding of brain proteins (10). C-to-U RNA editing is less prevalent than A-to-I RNA editing in humans (7). The few identified physiological C-to-U RNA editing targets include apolipoprotein B (apoB) pre-mRNA in intestinal cells and several recently validated, but uncharacterized mRNA targets; in an early study, 32 previously unknown APOBEC1 (apoB editing catalytic subunit 1) editing sites in the 3' untranslated regions (3' UTRs) of diverse mRNA transcripts were identified and validated (11). In addition, 56 novel editing sites in 54 intestinal mRNAs and 22 novel sites in 17 liver mRNAs in AU-rich segments of the 3' UTRs were also identified (12). Recently, 410 C-to-U RNA editing events across 275 transcripts were also found in bone marrow-derived macrophages, and nearly all (97\%) of these C-to-U events were detected in the 3' UTRs (13). C-to-U RNA editing of apoB pre-mRNA occurs at nucleotides 6666 and 6802 in the nucleus $(14,15)$. The C6666U editing event changes a glutamine codon
(CAA) to an in-frame translational stop codon (UAA). Thus, C6666-unedited apoB RNA generates a fulllength ApoB100 protein, whereas C6666U-edited apoB RNA yields a truncated ApoB48 protein $(48 \%$ of the full-length ApoB100 protein). ApoB100 is synthesized in the liver, whereas ApoB48 is generated in the small intestine. The tissue-specificity of apoB RNA editing in humans is due to the tissue-specific expression of apoB editing catalytic subunit 1 (APOBEC1), which is expressed solely in the small intestine. APOBEC-1 enzyme is a member of the cytidine deaminase (CDA) family that act on monomeric nucleoside and nucleotide substrates, and has ability to carry out the deamination from cytidine $(\mathrm{C})$ to uridine $(\mathrm{U})$ in pre-mRNA target. Both ApoB48 and ApoB100 proteins play important roles in regulation lipid metabolism (14). The C6802U editing event changes a threonine codon (ACA) to an isoleucine (AUA). The C6802U editing event occurs together with the $\mathrm{C} 6666 \mathrm{U}$ editing event; therefore, C6802U is detected in the mRNA but is not expressed in the truncated ApoB48 protein (15).

C-to-U RNA editing is dependent on cis-acting elements, as well as on the stoichiometric regulation of trans-acting factors within the macromolecular enzyme complex (editosome) responsible for targeted deamination. The cis-acting elements consist of 50 nucleotides (nt) flanking the edited cytidine containing a tripartite regulatory motif (the mooring sequence), which includes an 11-nt motif (UGAUCAGUAUA) located in an AU-rich sequence downstream of the edited base; these cis-acting elements combining with trans-acting factors are necessary and sufficient to carry out C-to-U RNA editing in vitro (16-20). The mooring sequence located at the $3^{\prime}$ of the edited cytidine is combined with a $5^{\prime}$ efficiency sequence to adopt a stable secondary structure that enhances specificity $(18,19)$. The core of the C-to-U editing editosome includes at least three proteins: APOBEC1 and two requisite cofactors, APOBEC1-complementation factor (ACF) and RNA-binding-motif-protein 47 (RBM47) (21). APOBEC1 is a member of the APOBEC family of RNA-specific cytidine deaminases. Similar to other members of the cytidine deaminase family, APOBEC1 contains a zinc-dependent deaminase domain important for cytidine deamination (22). APOBEC-1 forms homodimers in which specific amino acids within the APOBEC1 catalytic domain are bound to AU-rich sequences in the vicinity of the apoB pre-mRNA. This binding is weak and insufficient for mRNA association in vitro (23); therefore, the ACF cofactor (known as A1CF), a putative RNA-binding protein (RBP), is required. This cofactor binds the mooring sequence with high affinity and together with APOBEC1 forms the minimal editosome in in vitro experiments (21). ACF is a member of the Elav/HelN1/HuR family and contains multiple repeats of a single-stranded RNA-recognition motif (RRM). N- and C-terminal sequences flanking the 
multiple RRMs in ACF are necessary for its interaction with the APOBEC1 enzyme (24). Interestingly, while $\mathrm{ACF}$ knockout mice $\left(\mathrm{ACF}^{-/-}\right.$mutant mice) die early during gestation, editing efficiency is increased in $\mathrm{ACF}^{+-}$mutant mice, which is inconsistent with the fact that $\mathrm{ACF}$ is required for editosome activity in vitro. Although there is much biological evidence for ACF and APOBEC1-mediated C-to-U deaminase activity in vitro, there is as of yet no clear evidence that $\mathrm{ACF}$ is genetically required for apoB RNA editing in vivo. Thus, the role of ACF in the complex in vivo remains to be elucidated (25). Recently, a novel RBP (RBM47) was identified as another cofactor that is essential for C-to-U RNA editing in vivo (26). RBM47 can interact with APOBEC1 and ACF in the holoenzyme of the editosome and function with APOBEC1 in editing apoB transcripts. However, the functional consequences of the interaction between ACF and RBM47 in vivo remain unclear. Moreover, RBM47 can also substitute for ACF in the enzyme complex performing C-to- $\mathrm{U}$ RNA editing in vitro. Rbm $47^{\text {gt/gt }}$ mutant mice, which harbor a gene-trap mutant allele expressing $\beta$-geo instead of $R b m 47$, does not have detectable Rbm47 mRNA or RBM47 protein expression or Rbm47 activity and in the small intestines of these mice, the C-to-U RNA editing of apoB and four other C-to-U RNA editing targets (Sult1d1-Sulfotransferase 1 family member D1, Serinc1-Serine Incorporator 1, Casp6-Caspase 6 and 2010106E10Rik-RIKEN cDNA 2010106E10) was significantly reduced even though their expression levels were not changed. Therefore, RBM47 plays a critical role in C-to-U RNA editing and is a component of the editosome (26).

Interestingly, APOBEC3A (A3A), another member of the cytidine deaminase family that is structurally related to APOBEC1 and is expressed primarily in myeloid cells, including monocytes and macrophages, has been identified as a novel C-to-U RNA editing enzyme $(27,28)$. A3A functions in the inhibition of retrotransposons and several viruses including HIV1 (human immunodeficiency virus type 1), HTLV1 (human T-cell lymphotropic virus type 1), HPV (human papillomavirus), parvovirus, and hepatitis $B$. The transcripts of hundreds of genes, including implicated viral pathogenesis, Alzheimer's disease, peripheral blood monocytes exposed to hypoxia and/or interferons, and M1 (proinflammatory) macrophage differentiation, undergo site-specific C-to-U RNA editing $(27,28)$.

\section{C-to-U RNA editing in plants}

In addition to mammals, C-to-U RNA editing also takes place in plants. Although essential differences are known between C-to-U editing in mammals and $\mathrm{C}$-to-U editing in plants, it may be technically possible to modify or combine components of the plant RNAediting apparatus (especially the cytidine deaminase of plants) with guide RNA (complementary RNA sequence that specifically binds to target sequences) to replace the mammalian APOBEC1 enzyme in applications for human therapy. This approach would open up numerous applications in C-to- $\mathrm{U}$ editing if it is successfully developed. We therefore discuss C-to-U editing in plants, not only as a comparison with the process occurring in mammals, but also as an expected correlation. There are two main types of RNA editing occurring in plants: C-to-U RNA editing and, less frequently, U-to-C RNA editing. C-to-U RNA editing takes place in plant plastids and mitochondria (29-32). Both organelles contain their own circular genomes, which encode genes responsible for photosynthesis and respiration, respectively.

The circular genomes of plastids in higher plants are $120-130 \mathrm{~kb}$ nucleotides in size; it is estimated that 20 30 cytidines are converted to uridines, suggesting that the average editing frequency is approximately $0.02 \%$ in the plastid genome (33). Few mRNAs in plastids do not contain either start or stop codons; therefore, C-to-U editing enables the expression of functional proteins by generating start or stop codons and modifying amino acid sequences (34-36). In plastids, C-to-U RNA editing occurs in mRNAs but not in other RNA types, including transfer RNA (tRNA) and ribosome RNA (rRNA).

The average editing frequency in plant mitochondria is much higher than in plastid C-to-U RNA editing; for example, the total size of the mitochondrial genome in Arabidopsis thaliana (A. thaliana) is approximately $367 \mathrm{~kb}$, with only $30 \mathrm{~kb}$ consisting of genes encoding the subunits of respiratory chain complexes (37). However, 441 C-to-U RNA editing events have been identified in mitochondrial open reading frames (38). In contrast with plant plastids, C-to-U RNA editing of plant mitochondria occurs in both mRNA and tRNA, but not in rRNA. In mitochondria, C-to-U editing events occur in coding regions and less frequently in introns and other untranslated regions. These events restore and correct open reading frames to enable gene expression in plant mitochondria. Without C-to-U RNA editing, several respiratory chain proteins would not be produced and no functional mitochondria could be assembled and maintained in plant cells. In addition, C-to-U editing events in mitochondrial tRNAs are essential for restoring important base-pairings to correct the folding and processing of tRNA precursors $(39,40)$.

C-to-U RNA editing in plants involves various corresponding editing machineries and mechanisms. Moreover, it is clear that cis-elements adjacent to the editing sites are necessary to ensure $\mathrm{C}$-to- $\mathrm{U}$ editing specificity. Many studies have shown that, in contrast with mammalian cis-elements, the major cis-acting recognition elements in plants are located in the $5^{\prime}$ flanking region upstream of the editing sites, while the 3 ' flanking region makes a relatively small contribution to $\mathrm{C}$-to-U RNA editing efficiency. Cis-elements are 
required for C-to-U RNA editing in both plastids and mitochondria (41-45). In general, an upstream sequence of $<20$ nucleotides, and in some cases a downstream sequence of $<10$ nucleotides, suffice as cis-elements for RNA editing (46). Some components of transacting factors bind to cis-elements at the essential 5' flanking upstream region and, subsequently, the specific downstream cytidine is recognized and targeted as an editing site. Next, the RNA editing machinery is recruited to the editing site and enzymatic modification occurs, changing the targeted $\mathrm{C}$ to $\mathrm{U}$.

Several pentatricopeptide repeat (PPR) motifcontaining proteins have been identified as one part of the trans-acting factors required for C-to-U RNA editing in both chloroplasts and mitochondria. These PPR proteins have been characterized as site-specific recognition factors for a number of targeted cytidines that bind directly to cis-elements. PPR proteins consist of tandem arrays of degenerate 35-amino-acid repeats (the PPR motifs), which can vary in number from 2 to 30 (47-49). The PPR family can be divided into the P and PLS subfamilies, based on the PPR motif structure (50). The PPR family includes many members; approximately 450 family members exist in Arabidopsis including approximately 250 and $200 \mathrm{P}$ and PLS subfamily members, respectively (51). Members of the P subfamily contain a simple array of PPR motifs (P), whereas those of the PLS subfamily contain an array of triplets namely, long (L) and short (S) PPR-like motifs and canonical PPR motifs (P). Members of the PLS subfamily can be further divided into the PLS, E/E+ (Extended), and DYW (Aspartate-tyrosine-tryptophan) classes according to the different C-terminal domains $(50,52)$. Members of the PLS subfamily has been reported as trans-acting RNA editing factors in plants, whereas P subfamily members are associated with the RNA maturation process including the translational machinery. The PPR motif contains two anti-parallel $\alpha$-helices that interact with each other to generate a helix-turn-helix motif; a series of helix-turn-helix motifs is then combined into a super-helix that has a specific central groove. This central groove controls protein binding to specific cisacting element(s) on the targeted RNA with one PPR motif binding one nucleotide $(47,51)$. Arabidopsis species contain approximately $650 \mathrm{C}$-to-U RNA editing sites in two organelles. These sites are recognized by 200 members of the PLS subfamily, although it is possible that a single trans-acting factor could recognize more than two sites on average. For example, CRR22 (Chlororespiratory reduction22) and SOL2 recognize at least three cis-acting sites in the plastid and at least six sites in mitochondria, respectively $(53,54)$.

To date, it has been unclear how a single PPR protein recognizes multiple target sequences; however, recently, other accessory proteins such as RNA-editing factor-interacting protein (RIP, also known as multiple sites organellar RNA editing factors, or MORFs) (55-
58) and organelle RNA recognition motif factors (ORRMs) $(56,59)$ in the RNA editing apparatus have been identified. The MORF/RIP family in Arabidopsis includes 10 members. Of these, MORF2/RIP2 and MORF9/RIP9 have been reported in plastids, whereas MORF8/RIP1 has been identified in both plastids and mitochondria. RIP10 is encoded by a pseudogene, and the remaining family members have been reported to function in mitochondria. MORF proteins play an important role in C-to-U RNA editing, supporting an ordered spatial connection between PPR and other proteins. Another potential function of MORF proteins may contribute to site-specificity of the editing enzyme to the targeted $\mathrm{C}$ and may be associated with their ability to bind metal ions, such as cobalt (55). ORRMs contain an RRM at the C-terminus. The ORRM family has four major members. ORRM1 is a plastid editing factor, whereas ORRM2, ORRM3, and ORRM4 have been identified as mitochondrial RNA editing factors. The functions of ORRM proteins are unclear; however, they may play a similar role to the MORF factors. The interaction between PPR proteins and other trans-acting factors requires further investigation $(59,60)$.

The mechanism of C-to-U substitution editing in plastids and mitochondria is predicted to be very similar to that of apoB mRNA editing, and an APOBEC-1like cytidine deaminase enzyme may be involved in this process. At least eight cytidine deaminases have been identified in $A$. thaliana. One of these, $A$. thaliana cytidine deaminase 1 (At-CDA-1) has been characterized; however, this protein has no affinity for RNA and cannot perform RNA editing (61). These findings led to the hypothesis that a yet unidentified nuclear encoded protein may be exported to these organelles; to date, the components of the plant RNA editing apparatus are not fully understood.

\section{Enzymatic site-directed A-to-I RNA editing}

Therapeutic RNA editing or site-directed RNA editing is an approach that corrects mutated mRNA sequences in order to treat genetic diseases caused by point mutations (62). Using mRNA vs. DNA as a gene therapy target is advantageous because targeted mRNA will be replenished after treatment; thus, the applied changes are not maintained or passed on to the next generation as would occur by targeting DNA. Moreover, mRNA-targeted therapy does not alter the physiological level of the endogenous mRNA (63). The site-directed RNA editing machinery requires two main factors: a guide RNA component that specifically recognizes the sequence to be edited and another RNA editing enzyme component that performs the modifying activity (62). RNA editing is naturally prevalent in mammals; when it occurs in coding regions, it results in single amino acid substitutions, and when it occurs in noncoding regions, it leads to changes in RNA 
processing. Thus, if RNA editing could be targeted to a user-defined mRNA site, it would have great potential for the treatment of genetic diseases related to point mutations (64). Site-directed RNA editing includes site-directed C-to-U editing and A-to-I editing. Several site-directed RNA editing studies have been conducted recently; and all of which have successfully performed for manipulating site-directed A-to-I transitions $(63,64)$. C-to-U-transition editing has only been hypothetically reviewed. With further refinement of the technology, site-specific C-to-U editing may potentially be accomplished with similar strategies of enzymatic editing. Therefore, in this section, site-directed A-to-I editing will be described and explained in more detail.

A-to-I RNA editing converts adenosine (A) to inosine (I), which is read as guanosine $(\mathrm{G})$; thus, this conversion effectively results in a change from $A$ to $G$. When A-to-I editing occurs in an mRNA open reading frame, it can recode 12 of the 20 canonical amino acids into a substitute codon, or a start or stop codon (65). Well-known examples of transcripts that undergo A-to-I editing include the calcium-gated glutamate receptor (GluR-B) and the 5-hydroxytryptamine $2 \mathrm{C}$ receptor (5HT2c) (66). During RNA editing, the base conversions occur by hydrolytic deamination catalyzed by adenosine deaminase acting on RNA (ADAR) enzymes $(65,67)$. Members of the ADAR family can perform A-to-I RNA editing without additional cofactors in vitro (68). ADAR enzyme family includes three members (ADAR1, ADAR2, and ADAR3), which are highly conserved in mammalian cells. The A-to-I editing enzymatic activity of ADAR1 and ADAR2 has been demonstrated, whereas that of ADAR3 has not. ADAR enzymes consist of distinct domains that perform different functions. A catalytic domain containing conserved amino acid residues is situated at the C-terminus. This catalytic domain catalyzes the hydrolytic deamination of A to I. One-to-three repeats of the highly conserved dsRNA-binding domain (dsRBD) (approximately 65 amino acids) that bind(s) both perfect and imperfect RNA duplexes are found at the N-terminus (6).

An early therapeutic RNA editing study involved the design and synthesis of a synthetic complementary RNA oligonucleotide to direct the correction of a premature stop codon mutation in dystrophin RNA. The complementary RNA oligonucleotide was first inserted into the reaction. This complementary RNA oligonucleotide hybridizes perfectly with the targeted sequences that contain a premature stop codon and the hybrid activates the endogenous ADAR enzyme in nuclear extracts or in Xenopus embryos. Subsequently, the premature UAG stop codon is corrected to a UGG codon (Trp) both in vitro and in Xenopus embryos. Importantly, only complementary RNA oligonucleotide was used in the study, and the endogenously expressed enzymes were induced to nonspecifically edit the region (62).

To obtain specific site-directed RNA editing, the dsRBDs of human ADAR1 (hADAR1) or human ADAR2 (hADAR2) were replaced with an antisense RNA oligonucleotide-guide RNA turning them into guide RNA-dependent enzymes. The antisense RNA oligonucleotide has an important function in guiding the catalytic activity to a specific position on the mRNA and forming the secondary structure required for the highly efficient and selective editing of the single targeted adenosine. An RNA-binding protein that could be genetically encoded is required to link the hADAR deaminase domain and an antisense guide RNA oligonucleotide. The linkage between the hADAR deaminase domain and the RNA-binding protein, and the interaction between the antisense guide RNA and the RNA-binding protein should be small so that they do not interfere with the function of the deaminase domain or the guide RNA. Moreover, high-affinity binding is required to drive the editing process in the complex cellular environment (63). To date, only a few studies have been conducted to examine site-directed enzymatic RNA editing.

In one study, the deaminase domain of hADAR was coupled with the guide RNA by using an in vitro reaction. The covalent attachment between human $O^{6}$ alkylguanine-DNA-alkyltransferase (AGT) or SNAPtag and O6-benzylguanine (BG) was used to couple the deaminase domain of hADAR and the guide RNA. The SNAP-tag is a small protein, which is suitable for direct covalent interactions with $\mathrm{BG}$, resulting in the formation of a stable bond. The guide RNA-dependent deaminase was generated by substituting the N-terminal dsRBD with a SNAP-tag. The gene sequence encoding the hADAR 1 deaminase domain was combined with a sequence encoding a SNAP-tag in a single vector, resulting in the expression of a fusion consisting of the hADAR1 deaminase domain fused to a SNAP-tag. Next, synthesis of the BG-guide RNA was conducted by solid-phase peptide synthesis. The vector containing the hADAR1 deaminase domain SNAP-tag fusion and the BG-guide RNA were transfected into cells to generate a guide RNA-deaminase conjugate. The hADAR 1 deaminase domain and guide RNA were assembled by a rapid and specific single covalent bond between the SNAP-tag and BG moiety (Figure 1). After the guide RNA-deaminase conjugate was generated, it rapidly carried out site-directed specific RNA editing of the target mRNA, and the results showed the subsequent repair of a premature stop codon (UAG) into a tryptophan codon (UIG) in a fluorescent reporter gene, both in vitro and in human cells (293T) (69). Recently, the BG moiety was chemically masked using a light-sensitive 6-nitropiperonyloxymethyl (Npom) protection group, resulting in Npom-protected O6benzylguanine (Npom-BG). The Npom group has two regioisomers (N7 and N9); both ${ }^{\mathrm{N} 7} \mathrm{Npom}-\mathrm{BG}$ 


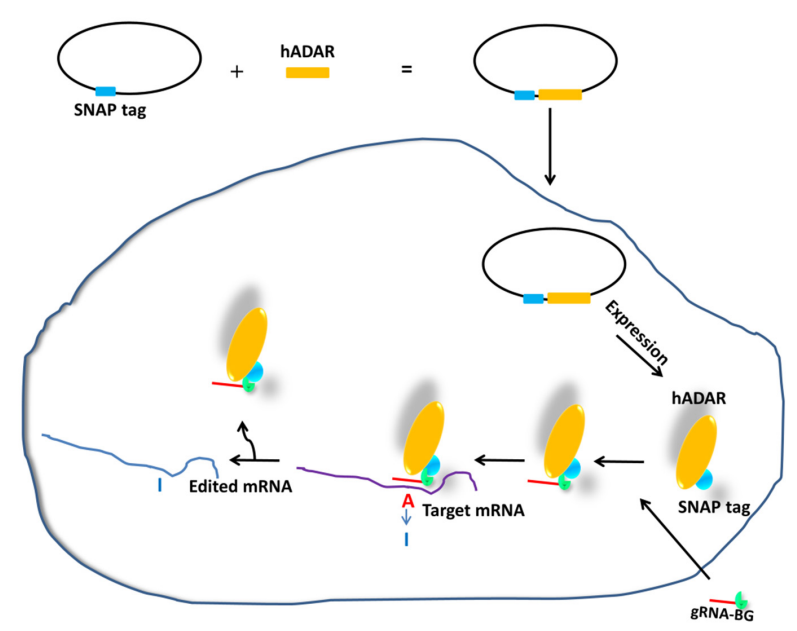

Figure 1. Schematic of experimental design. The catalytic domain of the human ADAR1 was fused with the C-terminus of a SNAP-tag. Next, the BG-modified gRNA was reacted with SNAP-deaminase to form guide RNA (gRNA)-deaminase conjugates. Finally, the gRNA-dependent deaminase carried out site-specific A-to-I RNA editing of a target mRNA (64).

and ${ }^{\mathrm{N} 9} \mathrm{Npom}-\mathrm{BG}$ efficiently decayed to release BG when irradiated with 365 -nm light using a common ultraviolet (UV) light table. Because the repair reaction (enzymatic site-directed A-to-I RNA editing) was dependent on the covalent attachment of guide RNA to the ADAR enzyme, the duration, location, and dosage of the editing reaction could be precisely controlled by triggering the assembly of the covalent RNA-protein conjugate (70).

In another study, the catalytic domain of hADAR2 was linked with antisense guide RNA using a naturally occurring binding reaction; $\lambda$-phage $\mathrm{N}$ protein-boxB $(\lambda \mathrm{N}$ peptide-boxB) was used to generate a linkage between the hADAR2 deaminase domain and the guide RNA. The $\lambda \mathrm{N}$ peptide consists of 22 amino acids, which were fused to the hADAR2 deaminase domain (DD), and the 17-nucleotide boxB containing a hairpin structure was combined with the guide RNA. The sequences that encode the hADAR2 deaminase domain were combined with the sequences encoding the $\lambda \mathrm{N}$ peptide in a single vector, resulting in expression of the $\lambda \mathrm{N}$-DD fusion protein. Next, the boxB sequences were linked with the guide RNA sequence in another vector. Both vectors were transcribed using an in vitro transcription kits. Then, the synthesized RNAs were directly injected into cells and, following translation, the $\lambda \mathrm{N}$ peptide rapidly bound to boxB with high affinity, thus generating a recombinant enzyme that could be directed efficiently and selectively to edit a single adenosine (Figure 2). In vitro assays have shown that this recombinant enzyme can successfully correct a premature termination codon in an mRNA encoding the cystic fibrosis transmembrane conductance regulator anion channel. Moreover, in Xenopus oocytes, the recombinant enzyme could also correct cystic fibrosis transmembrane

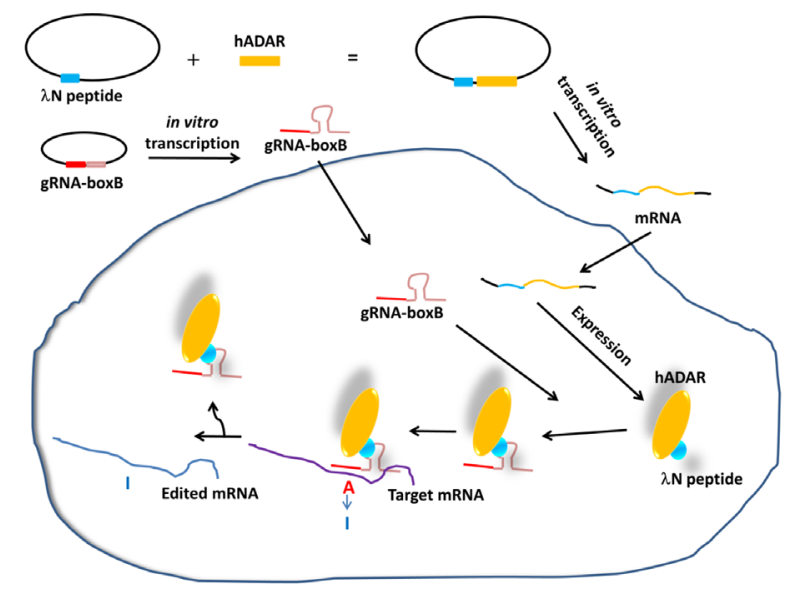

Figure 2. The overall strategy used for artificial sitedirected A-to-I RNA editing. A site-directed editase was generated by linking the hADAR2 deaminase domain with an antisense guide oligonucleotide through an interaction between the $\lambda$ phage $\mathrm{N}$ protein and the boxB RNA hairpin. Following the generation of the recombinant editase, the guide RNA (gRNA)-dependent deaminase conducted site-specific A-to-I RNA editing of the target mRNA (63).

conductance regulator mRNA, restore the full-length protein, and reestablish functional chloride currents across the plasma membrane; while in human cell lines it could correct a nonfunctional version of enhanced green fluorescent protein containing a premature termination codon (63).

Currently, site-directed enzymatic RNA editing is limited to mutations that can be corrected by recoding A-to-I; however, the same approach should be extended to cytidine deaminases that convert $\mathrm{C}$-to-U. If artificial site-directed enzymatic C-to-U RNA editing could be successfully completed, this enzymatic site-directed RNA editing approach could be applied to a wide variety of codons.

\section{Non-enzymatic site-directed C-to-U editing}

Non-enzymatic site-directed editing has prompted great interest recently because this method does not require the restrictive conditions dictated by enzymatic site-directed RNA editing. Fujimoto et al. were the first to report the application of this method for artificial C-to-U DNA and RNA editing. Their method involved the design and synthesis of 5-carboxyvinyl deoxyuridine nucleoside $\left({ }^{\mathrm{CV}} \mathrm{U}\right)$ - and 3-cyanovinylcarbazole nucleoside $\left({ }^{\mathrm{CNV}} \mathrm{K}\right)$ modified oligodeoxynucleotides (ODNs) for artificial DNA and RNA editing $(71,72)$ (Figure $3 \mathrm{~b}$ and Figure 4b). These modified ODNs were synthesized by standard phosphoramidite chemistry on a DNA synthesizer, with a post-modification procedure following ODN synthesis (71). In terms of the chemical structure, if deamination removes the amino group of cytidine (C) at the fourth position in the pyrimidine ring to form a ketone group, cytidine (C) converts to uridine (U).

These modified ODNs are covalently bound to 
A

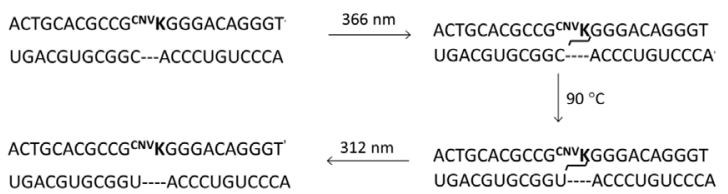

B

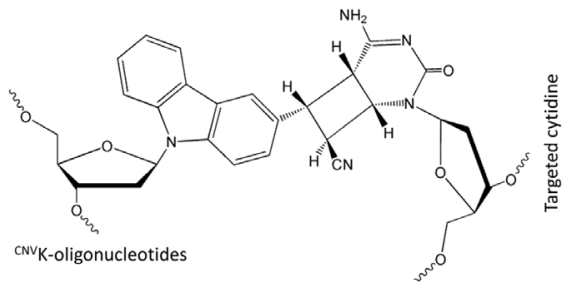

Figure 3. Schematic representation of an artificial C-to-U transition using ${ }^{\mathrm{CNV}} \mathrm{K}$ modified ODNs. (a) Steps of an artificial C-to-U transition. (b) Chemical crosslinking between the ${ }^{\mathrm{CNV}} \mathrm{K}$ photoreactive group and the targeted $\mathrm{C}$ in the target/ ODN heteroduplex (72).

the target DNA strand via a (2+2) photocycloaddition between the photoreactive nucleoside and deoxycytidine at a specific position in the target DNA following UV irradiation at $366 \mathrm{~nm}$. Next, the target cytidine undergoes photo-transition to uridine by heat-treatment; the target/ODN heteroduplex is UVirradiated at $312 \mathrm{~nm}$ leading to photosplitting and subsequent artificial editing with a C-to-U phototransition (71). The chemical mechanism underlying artificial RNA editing is not fully clear; however, it may utilize the same mechanism as artificial single-strand DNA editing. The developed photo-transition process also involves four main reactions. In the first step the modified ODNs and the target RNAs are hybridized; the hybridized modified ODNs and the target RNAs are then photo-crosslinked by UV irradiation at 366 $\mathrm{nm}$; next, deamination is achieved by heat treatment; and finally, the target RNAs and the modified ODNs are separated by photosplitting $(71,72)$ (Figure $3 a)$. The results of these studies demonstrated that nonenzymatic site-directed RNA editing exhibits high efficiency, without any side reactions occurring with ${ }^{\mathrm{CV}} \mathrm{U}$ - or ${ }^{\mathrm{CNV}} \mathrm{K}$-modified ODNs for many short singlestranded DNA and RNA targets, such as microRNA 27a and $27 \mathrm{~b}(71,72)$.

Different modified ODNs have different deamination efficiencies and working conditions. ${ }^{\mathrm{CV}} \mathrm{U}$-modified ODNs function at both physiological temperature $\left(37^{\circ} \mathrm{C}\right)$ and at non-physiological temperatures (high temperatures), while ${ }^{\mathrm{CNV}} \mathrm{K}$-modified ODNs function only at nonphysiological temperatures (high temperatures), and as with site-directed enzymatic RNA editing, mRNAs were used as targets because of the many advantages they afford compared with DNA targets, as previously shown. Therefore, in our study, we developed an applicable method for correcting genetic mutations such as Leigh syndrome in vitro and in other biological systems by using ${ }^{\mathrm{CV}} \mathrm{U}$-modified ODNs. As with previous methods, our phototherapy strategy involves the use of UVA

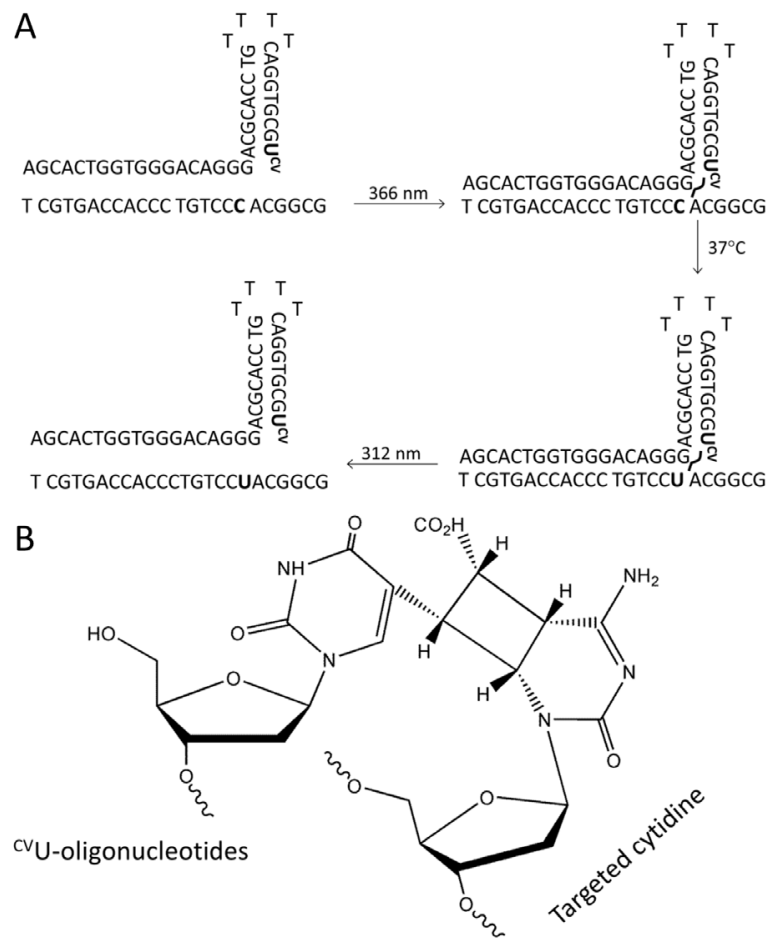

Figure 4. Schematic representation of an artificial C-to-U transition using ${ }^{\mathrm{CV}} \mathrm{U}$-modified ODNs. (a) Steps of an artificial C-to-U transition. (b) Chemical crosslinking between the ${ }^{\mathrm{CV}} \mathrm{U}$ photoreactive group and the targeted $\mathrm{C}$ in the target/ODN heteroduplex $(71,73)$.

radiation (366 $\mathrm{nm}$ ) to photo-crosslink ${ }^{\mathrm{CV}} \mathrm{U}$-containing ODNs and cytosine to mRNA targets. The deamination reaction is carried out during a heat-treatment step that is crucial for facilitating the $\mathrm{C}$-to- $\mathrm{U}$ substitution reaction. Finally, the cross-linked nucleotide is cleaved by photosplitting (UVB radiation; $312 \mathrm{~nm}$ ) (Figure 4a and Figure 5). UVB phototherapy is a commonly used treatment modality for psoriasis and other skin diseases. Although UVB has been associated with skin carcinogenesis, to date, no reports have demonstrated a correlation between UVB phototherapy and increased skin cancer risk. Therefore, UVB phototherapy was evaluated as a safe treatment modality (73).

To investigate photochemical editing, we used the T8993C mitochondrial DNA mutation of a Leigh syndrome patient as our initial model. Leigh syndrome is estimated to occur in approximately 1 of 40,000 live births. Leigh syndrome is a devastating, neurodegenerative disorder related to many types of point mutations; however, in $20 \%$ of patients, the disease is associated with the $8993 \mathrm{~T}>\mathrm{C}$ transversion in the mitochondrial DNA ATPase6 gene. The ATPase6 gene encodes a protein involved in the assembly of a subunit of respiratory chain complex V. To restore the mutant sequence to the wild-type sequence, nucleotide 8993 cytidine was converted into uridine in a Leigh syndrome patient. Single-strand (ss) DNA, either 72 or 731 nucleotides in length, was first used as DNA targets to optimize the non-enzymatic site-directed RNA editing 

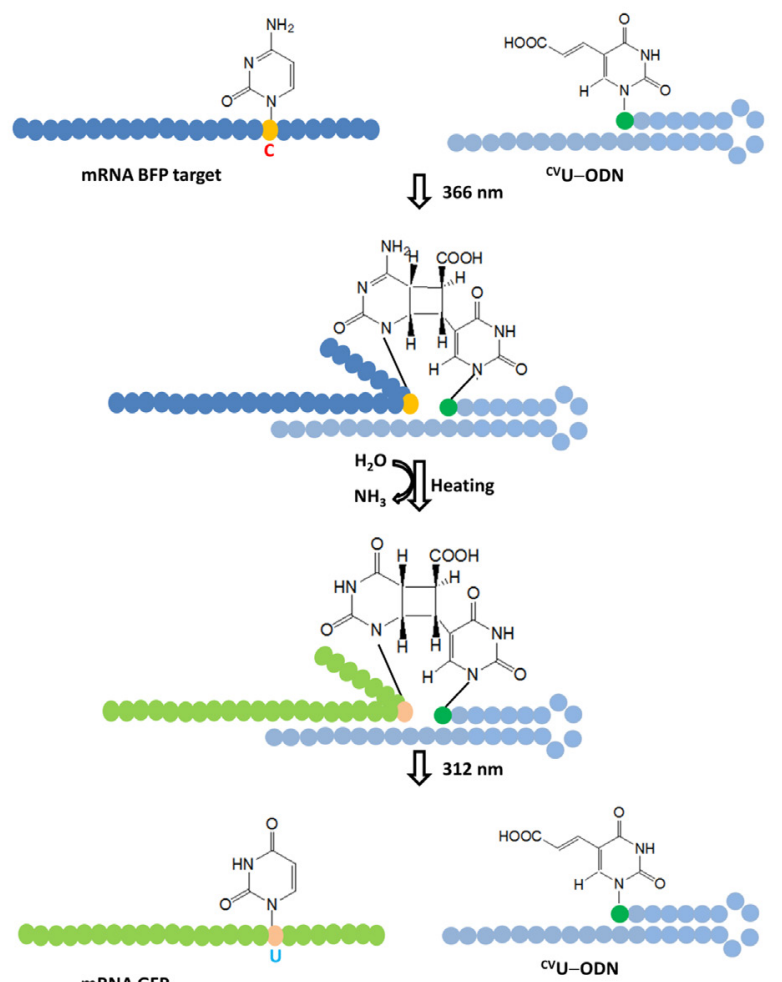

mRNAGFP

Figure 5. Schematic representation of the four main reactions of the photo-transition process. First, hybridization occurs between the modified ODN and the target RNA. Second, photo-crosslinking between the interacting ODNs is achieved by UV irradiation at $366 \mathrm{~nm}$. Third, heat treatment is performed to drive deamination. Fourth, the target RNA and modified ODN are separated by photosplitting (312 nm UV irradiation). Subsequently, artificial editing occurs with a C-to-U photo-transition $(71,73)$.

reaction. Then, the full-length 823-nt RNA and total RNA were used.

Site-directed deamination was performed as described in Figure 5. Base substitution from $\mathrm{C}$ to $\mathrm{U}$ was confirmed by PCR followed by restriction fragment length polymorphism analysis. Mva I could cut the wild-type "CCTGG" sequence, but not the mutant "CCCGG" sequence. Our results demonstrated that successful sequence-specific photochemical base substitution was achieved with various targets, including synthetic single-stranded 72 nucleotide (ss72-nt), ss731-nt, RNA 823-nt, and total RNA from the patient's cells, as targets. Our results showed that approximately $10 \%$ of the full-length mRNA was targeted for in vitro deamination under physiological temperatures (74).

Cells from the Leigh syndrome patient grew slowly and had difficulties taking up exogenous ODNs; therefore, blue fluorescent protein (BFP), a derivative of green fluorescent protein (GFP), was used as a new and more suitable model. BFP differs from GFP by a single nucleotide; therefore, the nt-199 conversion of cytidine to uridine transforms BFP to GFP. Sitedirected deamination was also performed as previously described. Briefly, the responsive ${ }^{\mathrm{CV}} \mathrm{U}$-modified ODNs were annealed to the mRNA BFP targets. The mutated cytidine was cross-linked with the responsive ODN by UV radiation $(366 \mathrm{~nm})$ and then received heattreatment. Finally, the cross-linked nucleotide is cleaved by the photosplitting operation (312 nm UV). A C-to-U photo-transition product (mRNA GFP) and ${ }^{\mathrm{CV}} \mathrm{U}$ ODNs were separated after the site-directed deamination process (Figure 5). To confirm the base substitution, PCR-restriction fragment length polymorphism and spectrofluorometry were used. BtgI, a restriction enzyme that can digest "CCACGG" in the coding sequence of BFP, but not "CTACGG" in GFP, was suitable for assessing the BFP-to-GFP transition. Our results showed that site-directed photochemical base substitution was successfully achieved using synthetic single-stranded 100 nucleotide (ss100-nt) and in vitro-synthesized full-length BFP mRNA targets. Approximately 10\% efficiency for the $\mathrm{C} 199 \mathrm{U}$ transition was exhibited under a physiological temperature $\left(37^{\circ} \mathrm{C}\right)$. Although the efficiency was not high, this result constitutes a first step towards using non-enzymatic site-directed transition to restore mutated mRNAs (73).

The structure and sequence of ${ }^{\mathrm{CV}} \mathrm{U}$-modified ODNs are key features affecting the biological functions of ODNs. To investigate the relationship between the ${ }^{\mathrm{CV}} \mathrm{U}$ - modified sequences and deamination efficiency, a series of ODNs, including $8{ }^{\mathrm{CV}} \mathrm{U}$-modified ODNs, was subjected to non-enzymatic site-directed C-to-U RNA editing. About structure, the ${ }^{\mathrm{CV}} \mathrm{U}$-modified ODNs contain three sections: the complementary section, the hairpin loop, and the 5'-terminal photoresponsive nucleobase ${ }^{\mathrm{CV}} \mathrm{U}$. The complementary section plays an important role in anchoring and site-directing the ODNs to their target; the hairpin loop allows ODNs easy access to cells and increases stability. The 5'-terminal photoresponsive nucleobase- ${ }^{\mathrm{CV}} \mathrm{U}$ constitutes the photoreactive group. Because the 5'-terminal photoresponsive nucleobase ${ }^{\mathrm{CV}} \mathrm{U}$ was the same in all ${ }^{\mathrm{CV}} \mathrm{U}$-modified ODNs, optimization of the complementary section length and hairpin loop length could be manipulated to control deamination efficiency. First, to investigate the structure-deamination efficiency relationship, the length of the hairpin loop was fixed, and the length of the complementary region was changed. In this case, the deamination efficiency depended on the length of complementary region. The lengths of the hairpin structure were set at 7, 9, and 11 $\mathrm{nt}$, and the lengths of the complementary regions used ranged from 10 to $22 \mathrm{nt}$ in length. Next, to evaluate the effects of the hairpin loop length on the deamination efficiency, the length of complementary region was fixed at $10 \mathrm{nt}$, and the lengths of hairpin structure used were set at 7, 9, and $11 \mathrm{nt}$. Photochemical base substitution was performed as described (Figure 5). Using a transition from the cytosine (C) of BFP gene to the uridine (U) of GFP gene at position 199 as a model, we were able to demonstrate that the structural sequence-dependent deamination efficiency was very 


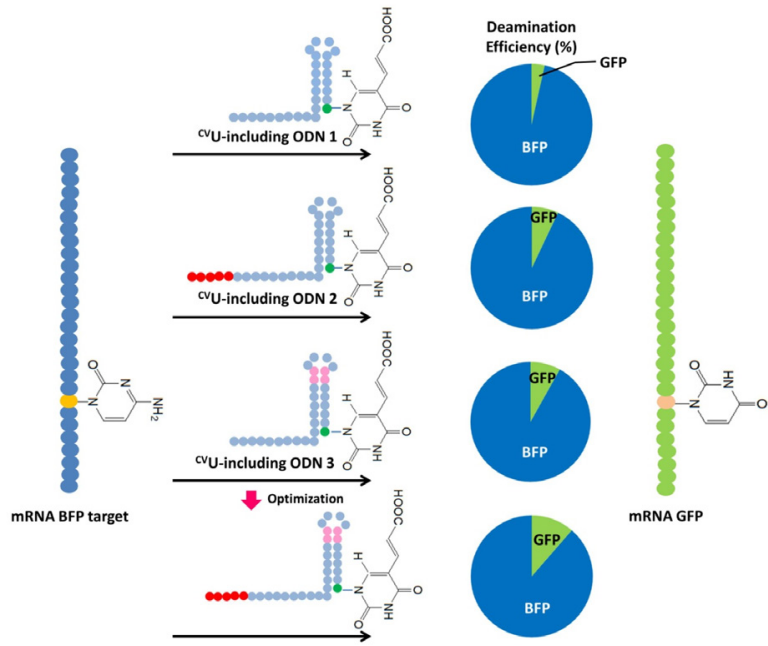

Figure 6. Schematic representation of the dependence of the deamination efficiency on the lengths of the complimentary effector ODN sequence and hairpin loop, which were determined to optimize the ${ }^{\mathrm{CV}} \mathrm{U}$-modified ODN structure. In our study, the deamination efficiency reached its maximum values with a complementary sequence length slightly more than $14 \mathrm{nt}$ and a hairpin loop length of $9 \mathrm{nt}(75)$.

tight in vitro. The optimum length of the hairpin was 9 nt, and the optimum length of complementary sequence was 15-17 nt (75) (Figure 6).

The advantage of non-enzymatic site-directed RNA editing is that the reaction can occur independently of conditions required for enzymatic activity; however, the disadvantage of this approach is low deamination efficiency. Further studies will be required to create other photoreactive groups to improve the deamination efficiency.

\section{Conclusion}

C-to-U RNA editing is an interesting biological phenomenon; however, to date its underlying enzymatic and complementary components are not fully understood. Further studies are required to elucidate the components and mechanisms of C-to-U RNA editing in both mammals and plants because this process holds great potential for the correction of $\mathrm{T}>\mathrm{C}$ or $\mathrm{A}>\mathrm{G}$ genetic mutations. Although enzymatic site-directed $\mathrm{C}$-to-U RNA editing has not yet to be successfully performed, in theory, it involves the same strategy already used successfully for enzymatic A-to-I site-directed RNA editing; thus, in the immediate future, enzymatic sitedirected C-to-U editing should be evaluated further in practical applications. In addition, non-enzymatic site-directed C-to-U RNA editing was successfully performed even though it has a lower deamination efficiency. The next steps for non-enzymatic sitedirected RNA editing involve the development of other modified ODNs to improve and increase the deamination efficiency at a physiological temperature. In the future, site-directed C-to-U RNA editing including, enzymatic C-to-U editing and non-enzymatic C-to-U editing, promises to effectively correct a variety of human diseases related to $\mathrm{T}>\mathrm{C}$ or $\mathrm{A}>\mathrm{G}$ genetic mutations.

\section{Acknowledgements}

We wish to thank Editage (www.editage.jp) for English language editing. This research was supported in part by a Grant-in-Aid for Scientific Research from the Japan Society for the Promotion of Science in Japan (25290072 and 26670167 to T.T.).

\section{References}

1. Gott JM, Emeson RB. Functions and mechanisms of RNA editing. Annu Rev Genet. 2000; 34:499-531.

2. Chen SH, Habib G, Yang CY, Gu ZW, Lee BR, Weng SA, Silberman SR, Cai SJ, Deslypere JP, Rosseneu M, Gotto AM, Li WH, Chan L. Apolipoprotein B-48 is the product of a messenger RNA with an organ-specific inframe stop codon. Science. 1987; 238:363-366.

3. Powell LM, Wallis SC, Pease RJ, Edwards YH, Knott TJ, Scott J. A novel form of tissue-specific RNA processing produces a polipoprotein-B48 in intestine. Cell. 1987; 50:831-840.

4. Freyer R, Kiefer-Meyer MC, Kössel H. Occurrence of plastid RNA editing in all major lineages of land plants. Proc Natl Acad Sci USA. 1997; 94:6285-6290.

5. Zanlungo S, Béqu D, Quiñones V, Araya A, Jordana X. RNA editing of a pocytochrome $b$ (cob) transcripts in mitochondria from two genera of plants. Curr Genet. 1993; 24:344-348.

6. Nishikura K. Functions and regulation of RNA editing by ADAR deaminases. Annu Rev Biochem. 2010; 79:321-349.

7. Smith HC, Bennett RP, Kizilyer A, McDougall WM, Prohaska KM. Functions and regulation of the APOBEC family of proteins. Semin Cell Dev Biol. 2012; 23:258268.

8. Bazak L, Haviv A, Barak M, Jacob-Hirsch J, Deng P, Zhang R, Isaacs FJ, Rechavi G, Li JB, Eisenberg E, Levanon EY. A-to-I RNA editing occurs at over a hundred million genomic sites located in a majority of human genes. Genome Res. 2013; 24:365-376.

9. Ramaswami G, Lin W, Piskol R, Tan MH, Davis C, Li JB. Accurate identification of human alu and non-alu RNA editing sites. Nat Methods. 2012; 9:579-581.

10. Li JB, Church GM. Deciphering the functions and regulation of brain-enriched A-to-I RNA editing. Nat Neurosci. 2013; 16:1518-1522.

11. Rosenber BR, Hamilton CE, Mwangi MM, Dewell S, Papavasiliou FN. Transcriptome-wide sequencing reveals numerous APOBEC1 mRNA-editing targets in transcript 3' UTRs. Nat Struct Mol Biol. 2011; 18:230-236.

12. Blanc V, Park E, Schaefer S, Miller M, Lin Y, Kennedy S, Billing AM, Hamidane HB, Graumann J, Mortazavi A, Nadeau JH, Davidson NO. Genome-wide identification and functional analysis of Apobec-1-mediated C-to-U RNA editing in mouse small intestine and liver. Genome Biol. 2014; 15:R79.

13. Harjanto D, Papamarkou T, Oates CJ, Rayon-Estrada V, Papavasiliou FN, Papavasiliou A. RNA editing 
generates cellular subsets with diverse sequence within populations. Nat Commun. 2016; 7:12145.

14. Blanc V, Davidson NO. Mouse and other rodent models of $\mathrm{C}$ to U RNA editing. Methods Mol Biol. 2011; 718:121-135.

15. Navaratnam N, Patel D, Shah RR, Greeve JC, Powell LM, Knott TJ, Scott J. An additional editing site is present in apolipoprotein B mRNA. Nucleic Acids Res. 1991; 19:1741-1744.

16. Backus JW, Smith HC. Three distinct RNA sequence elements are required for efficient apolipoprotein B (apoB) RNA editing in vitro. Nucleic Acids Res. 1992; 20:6007-6014.

17. Backus JW, Smith HC. Apolipoprotein B mRNA sequences $3^{\prime}$ of the editing site are necessary and sufficient for editing and editosome assembly. Nucleic Acids Res. 1991; 19:6781-6786.

18. Hersberger M, Innerarity TL. Two efficiency elements flanking the editing site of cytidine 6666 in the apolipoprotein B mRNA support mooring-dependent editing. J Biol Chem. 1998; 273:9435-9442.

19. Nakamuta M, Tsai A, Chan L, Davidson NO, Teng BB. Sequence elements required for apolipoprotein B mRNA editing enhancement activity from chicken enterocytes. Biochem Biophys Res Commun. 1999; 254:744-750.

20. Shah RR, Knott TJ, Legros JE, Navaratnam N, Greeve JC, Scott J. Sequence requirements for the editing of apolipoprotein B mRNA. J Biol Chem. 1991; 266:1630116304.

21. Mehta A, Kinter MT, Sherman NE, Driscoll DM. Molecular cloning of apobec-1 complementation factor, a novel RNA-binding protein involved in the editing of apolipoprotein B mRNA. Mol Cell Biol. 2000; 20:18461854.

22. Navaratnam N, Bhattacharya S, Fujino T, Patel D, Jarmuz AL, Scott J. Evolutionary origins of apoB mRNA editing: Catalysis by a cytidine deaminase that has acquired a novel RNA-binding motif at its active site. Cell. 1995; 81:187-195.

23. MacGinnitie AJ, Anant S, Davidson NO. Mutagenesis of apobec-1, the catalytic subunit of the mammalian apolipoprotein B mRNA editing enzyme, reveals distinct domains that mediate cytosine nucleoside deaminase, RNA binding, and RNA editing activity. J Biol Chem. 1995; 270:14768-14775.

24. Wedekind JE, Dance GS, Sowden MP, Smith HC. Messenger RNA editing in mammals: New members of the APOBEC family seeking roles in the family business. Trends Genet. 2003; 19:207-216.

25. Blanc V, Henderson JO, Newberry EP, Kennedy S, Luo J, Davidson NO. Targeted deletion of the murine apobec-1 complementation factor (acf) gene results in embryonic lethality. Mol Cell Biol. 2005; 25:7260-7269.

26. Fossat N, Tourle K, Radziewic T, Barratt K, Leibhold D, Studdert JB, Power M, Jones V, Loebel DAF, Tam PP. $\mathrm{C}$ to $\mathrm{U}$ RNA editing meditated by APOBEC1 requires RNA-binding protein RBM47. EMBO Rep. 2014; 15:903-910.

27. Sharma S, Patnaik SK, Taggart RT, Kannisto ED, Enriquez SM, Gollnick P, Baysal BE. APOBEC3A cytidine deaminase induces RNA editing in monocytes and macrophages. Nat Commun. 2015; 6:6881.

28. Sharma S, Patnaik SK, Kemer Z, Baysal BE. Transient overexpression of exogenous APOBEC3A causes C-to-U RNA editing of thousands of genes. RNA Biol. 2016; 1-8.
29. Covello PS, Gray MW. RNA editing in plant mitochondria. Nature. 1989; 341:662-666.

30. Gualberto JM, Lamattina L, Bonnard G, Weil JH, Grienenberger JM. RNA editing in wheat mitochondria results in the conversation of protein sequences. Nature. $1989 ; 341: 660-662$.

31. Hiesel R, Wissinger B, Schuster W, Brennicke A. RNA editing in plant mitochondria. Science. 1989; 246:16321634.

32. Maier MR, Neckermann K, Igloi GL, Kössel H. Complete sequence of the maize chloroplast genome: Gene content, hotspots of divergence and fine tuning of genetic information by transcript editing. J Mol Biol. 1995; 251:614-628.

33. Bailey-Serres J, Rochaix JD, Wassenegger M, Filipowicz W. Plant, their organelles, viruses and transgenes reveal the mechanisms and relevance of post-transcriptional processes Leysin, VD, Switzeland, February 25-28, 1999. EMBO J. 1999; 18:5153-5158.

34. Kotera E, Tasaka M, Shikanai T. A pentatricopetide repeat protein is essential for RNA editing in chloroplasts. Nature. 2005; 433:326-330.

35. Sasaki Y, Kozaki A, Ohmori A, Iguchi H, Nagano Y. Chloroplast RNA editing required for functional acetyl-CoA carboxylase in plants. J Biol Chem. 2001; 276:3937-3940.

36. Wintz $\mathrm{H}$, Hanson MR. A termination codon is created by RNA editing in the petunia atp9 transcript. Curr Genet. 1991; 19:61-64.

37. Unseld M, Marienfeld JR, Brandt P, Brennicke A. The mitochondrial genome of Arabidopsis thaliana contains 57 genes in 366,924 nucleotides. Nat Genet. 1997; 15:57-61.

38. Giegé P, Brennicke A. RNA editing in Arabidopsis mitochondria effects $441 \mathrm{C}$ to $\mathrm{U}$ changes in ORFs. PNAS. 1999; 96:15324-15329.

39. Marchfelder A, Binder S. Plastid and plant mitochondrial RNA processing and RNA stability. In: Molecular Biology and Biotechnology of Plant Organelles (Daniell H, Chase CD, eds.). Springer, Dordrecht, 2004; pp. 261294.

40. Takenaka M, Verbitskiy D, van der Merwe JA, Zehrmann A, Brennicke A. The process of RNA editing in plant mitochondria. Mitochondrion. 2008; 8:35-46.

41. Chaudhuri S, Carrer H, Maliga P. Site-specific factor involved in the editing of the psbL mRNA in tobacco plastids. EMBO J. 1995; 14:2951-2957.

42. Bock R, Hermann M, Kössel H. In vivo dissection of cisacting determinants for plastid RNA editing. EMBO J. 1996; 15:5052-5059.

43. Chaudhuri S, Maliga P. Sequences directing $C$ to $U$ editing of the plastid psbL mRNA are located within a 22 nucleotide segment spanning the editing site. EMBO J. 1996; 15:5958-5964.

44. Bock R, Hermann M, Fuchs M. Identification of critical nucleotide positions for plastid RNA editing site recognition. RNA. 1997; 3:1194-1200.

45. Hermann M, Bock R. Transfer of plastid RNA-editing activity to novel sites suggests a critical role for spacing in editing-site recognition. Proc Natl Acad Sci U S A. 1999; 96:4856-4861.

46. Shikanai T. RNA editing in plant organelles: Machinery, physiological function and evolution. Cell Mol Life Sci. 2006; 63:698-708.

47. Small ID, Peeters N. The PPR motif - a TPR-related 
motif prevalent in plant organellar proteins. Trends Biochem Sci. 2000; 25:46-47.

48. Aubourg S, Boudet N, Kreis M, Lecharny A. In Arabidopsis thaliana, $1 \%$ of the genome codes for a novel protein family unique to plants. Plant Mol Biol. 2000; 42:603-613.

49. Lurin C, Andrés C, Aubourg S, et al. Genome-wide analysis of Arabidopsis pentatricopeptide repeat proteins reveals their essential role in organelle biogenesis. Plant Cell. 2004; 16:2089-2103.

50. Shikanai T, Endo T, Hashimoto T, Yamada Y, Asada K, Yokota A. Directed disruption of the tobacco ndhB gene impairs cyclic electron flow around photosystem I. Proc Natl Acad Sci USA. 1998; 95:9705-9709.

51. Schmitz-Linneweber C, Small I. Pentatricopeptide repeat proteins: A socket set for organelle gene expression. Trends Plant Sci. 2008; 13:663-670.

52. Barkan A, Small I. Pentatricopeptide repeat proteins in plants. Annu Rev Plant Biol. 2014; 65:415-442.

53. Okuda K, Chateigner-Boutin AL, Nakamura T, Delannoy E, Sugita M, Myouga F, Motohashi R, Shinozaki K, Small I, Shikanai T. Pentatricopeptide repeat proteins with the DYW motif have distinct molecular functions in RNA editing and RNA cleavage in Arabidopsis chloroplasts. Plant Cell. 2009; 21:147-156.

54. Zhu Q, Dugardeyn J, Zhang C, Takenaka M, Kühn K, Craddock C, Smalle J, Karampelias M, Denecke J, Peters J, Gerats T, Brennicke A, Eastmond P, Meyer EH, Van Der Straeten D. SLO2, a mitochondrial pentatricopeptide repeat protein affecting several RNA editing sites, is required for energy metabolism. Plant J. 2012; 71:836-849.

55. Bentolila S, Oh J, Hanson MR, Bukowski R. Comprehensive high-resolution analysis of the role of an Arabidopsis gene family in RNA editing. PLoS Genet. 2013; 9:e1003584.

56. Bentolila S, Heller WP, Sun T, Babina AM, Friso G, vanWijk KJ, Hanson MR. RIP1, a member of an Arabidopsis protein family, interacts with the protein RARE1 and broadly affects RNA editing. Proc Natl Acad Sci U S A. 2012; 109:E1453-1461.

57. Takenaka M, Zehrmann A, Verbitskiy D, Kugelmann M, Hartel B, Brennicke A. Multiple organellar RNA editing factor (MORF) family proteins are required for RNA editing in mitochondria and plastids of plants. Proc Natl Acad Sci U S A. 2012; 109:5104-5109.

58. Boussardon C, Avon A, Kindgren P, Bond CS, Challenor M, Lurin C, Small I. The cytidine deaminase signature $\mathrm{HxE}(\mathrm{x}) \mathrm{n}$ CxxC of DYW1 binds zinc and is necessary for RNA editing of ndhD-1. New Phytol. 2014; 203:10901095.

59. Sun T, Germain A, Giloteaux L, Hammani K, Barkan A, Hanson MR, Bentolila S. An RNA recognition motifcontaining protein is required for plastid RNA editing in Arabidopsis and maize. Proc Natl Acad Sci USA. 2013; 110:E1169-1178.

60. Shi X, Bentolila S, Hanson MR. Organelle RNA recognition motif-containing (ORRM) proteins are plastid and mitochondrial editing factors in Arabidopsis. Plant Signal Behav. 2016; 11:e1167299.
61. Chen M, Herde M, Witte CP. Of the nine cytidine deaminase-like genes in Arabidopsis, eight are pseudogenes and only one is required to maintain pyrimidine homeostasis in vivo. Plant Physiol. 2016; 171:799-809.

62. Woolf TM, Chase JM, Stinchcomb DT. Toward the therapeutic editing of mutated RNA sequences. Proc Natl Acad Sci U S A. 1995; 92:8298-8302.

63. Montiel-Gonzalez MF, Vallecillo-Viejo I, Yudowski GA, Rosenthal JJC. Correction of mutations within the cystic fibrosis transmembrane conductance regulator by sitedirected RNA editing. Proc Natl Acad Sci U S A. 2013; 110:18285-18290

64. Schneider MF, Wettengel J, Hoffmann PC, Stafforst T. Optimal guideRNAs for re-directing deaminase activity of hADAR1 and ADAR2 in trans. Nucleic Acids Res. 2014; 42:e87.

65. Bass BL. RNA editing by adenosine deaminases that act on RNA. Annu Rev Biochem. 2002; 71:817-846.

66. Maas S, Rich A. Changing genetic information through RNA editing. Bioessays. 2000; 22:790-802.

67. Smith HC, Sowden MP. Base-modification mRNA editing through deamination: The good, the bad and the unregulated. Trends Genet. 1996; 12:418-424.

68. Chen CX, Cho DS, Wang Q, Lai F, Carter KC, Nishikura $\mathrm{K}$. A third member of the RNA-specific adenosine deaminase gene family, ADAR3, contains both singleand double-stranded RNA binding domains. RNA. 2000; 6:755-767.

69. Vogel P, Schneider MF, Wettengel J, Stafforst T. Improving site-directed RNA editing in vitro and in cell culture by chemical modification of the guideRNA. Angew Chem Int Ed. 2014; 53:6267-6271.

70. Hanswillemenke A, Kuzdere T, Vogel P, Jekely G, Stafforst T. Site-directed RNA editing in vivo can be triggered by the light-driven assembly of an artificial riboprotein. J Am Chem Soc. 2015; 137:15875-15881.

71. Fujimoto K, Matsuda S, Yoshimura Y, Matsumura T, Hayashi M, Saito I. Site-specific transition of cytosine to uracil via reversible DNA photoligation. Chem Commun. 2006; 30:3223-3225.

72. Fujimoto K, Konishi-Hiratsuka K, Sakamoto T, Yoshimura Y. Site-specific photochemical RNA editing. Chem Commun. 2010; 46:7545-7547.

73. Vu LT, Nguyen TTK, Alam S, Sakamoto T, Fujimoto K, Suzuki H, Tsukahara T. Change from blue fluorescent protein to green fluorescent protein using chemical RNA editing as a novel strategy in genetic restoration. Chem Biol Drug Des. 2015; 86:242-1252.

74. Vu LT, Ooka Y, Alam S, Suzuki H, Fujimoto K, Tsukahara T. Chemical RNA editing as a possibility novel therapy for genetic disorders. IJAC. 2012; 2:237241.

75. Vu LT, Nguyen TTK, Md Thoufic AA, Suzuki H, Tsukahara T. The relationship between structures and deamination efficiency of carboxyvinyldeoxyuridine ODNs on chemical RNA editing for genetic restoration. Chem Biol Drug Des. 2016, 87:583-593.

(Received March 2, 2017; Revised March 29, 2017; Accepted April 11, 2017) 